\title{
Wilson's Disease Presenting with Generalized Tonic-Clonic Seizure and Cerebellar Dysfunction
}

\author{
Aziz Rahman Rasib (D) \\ Aemal Aziz Jabarkhil \\ Mohammad Faiq Sediqi ${ }^{2,3}$ \\ Ahmad Irshad Mansoor ${ }^{1,4}$ \\ Abdullah Asady (iD ${ }^{5}$ \\ 'Department of Neuropsychiatry, Kabu \\ University of Medical Sciences, Kabul, \\ I00I, Afghanistan; ${ }^{2}$ Department of \\ Histology, Kabul University of Medical \\ Sciences, Kabul, I00I, Afghanistan; \\ ${ }^{3}$ Department of Basic Medical Sciences, \\ International Islamic University Malaysia, \\ Kuantan, 25200, Malaysia; ${ }^{4}$ Division of \\ Psychology \& Systems Sciences, Institute \\ of Psychiatry, Psychology \& Neuroscience, \\ King's College London and London School \\ of Hygiene and Tropical Medicine, \\ London, UK; ${ }^{5}$ Department of \\ Microbiology, Kabul University of Medical \\ Sciences, Kabul, I00I, Afghanistan
}

\begin{abstract}
Wilson's disease (WD) is a rare inherited impaired copper metabolism with diverse clinical pictures dominated by hepatic and neurologic manifestations. We report the case of a 14-year-old female patient who attended the Department of Neuropsychiatry at Ali Abad Teaching Hospital, Kabul, Afghanistan, with generalized tonic-clonic seizure and cerebellar dysfunction. The patient was initially diagnosed as encephalitis and epilepsy and finally diagnosed with WD based on the clinical and laboratory findings. After 6 months of follow-up, the patient showed substantial clinical recovery.
\end{abstract}

Keywords: Wilson's disease, copper accumulation, KF rings, seizure, cerebellar dysfunction

\section{Introduction}

Wilson's disease (WD) is an infrequent autosomal recessive disturbance of copper metabolism which happens as a result of mutations in the copper-transporting ATP7B gene. ${ }^{1}$ Increased free copper levels in different organs cause injury to them, with broad-ranging clinical manifestations dominated by signs of liver and brain injury, especially of the basal ganglia and cerebellum. ${ }^{2}$ Abnormal movements such as tremor, dystonia, bradykinesia, and chorea, accompanied with difficulty swallowing, difficulty speaking and poor articulation, and excessive salivation are the chief neurologic manifestations. ${ }^{3}$ Apart from movement disorders, neurologic signs of WD are uncommon and include neuropathy, autonomic system dysfunction, headaches, and epilepsy. ${ }^{3}$ Epilepsy may be one of the earliest presentations of $\mathrm{WD}$, sometimes years before other neurological symptoms appear. ${ }^{4}$ The frequency and importance of epilepsy in WD patients were first described by Dening et al in $1988 .{ }^{4}$ In this study, the authors found seizures in $6.2 \%$ of 200 patients (in England) - 10 times more than the frequency of seizures in general, healthy population. ${ }^{3}$ Neurologic manifestations of WD are revealed at an older age compared to hepatic manifestations. They are commonly found in patients with misdiagnosed hepatic disease, in those with asymptomatic hepatic impairment, in the case of non-adherence to de-coppering therapy, or with treatment failure. ${ }^{5}$ In view of its diverse presentations, misdiagnosis is not unusual. ${ }^{6}$

\section{Case Presentation}

A 14-year-old female patient came to the Department of Neuropsychiatry at Ali Department of Neuropsychiatry, Kabul University of Medical Sciences, Kabul,

I00I, Afghanistan

Tel +93797089250

Email azizrahmanrasib@gmail.com
Abad Teaching Hospital, Kabul, Afghanistan with generalized tonic-clonic seizure, ataxia, intention tremor, and disturbance of gait and speech. During investigation, it was noted that she had suffered from seizure attacks for one year. It was 
accompanied by ataxia, intention tremor, and disturbances of gait and speech in the last nine months. The symptoms had started insidiously and progressed gradually over time. The situation had worsened in the last month to the extent that she was not able to perform her daily activities without support. Throughout this period, she had been hospitalized under various conditions, including encephalitis and epilepsy, and had taken various medicines without any improvement.

Nothing significant was found in her family history. On general physical examination, the patient was alert, cooperative, and oriented. No abnormal finding was noted in other systems except for a dark ring in the cornea of both eyes as shown in Figure 1. On neurologic examination, ataxia, intention and resting tremor, dysarthria, tandem gait, dysmetria, and abnormal finger to finger test were present.

Lab investigations including complete blood count, liver function test, and renal function test were within normal ranges. Hepatitis B surface antigen and hepatitis $\mathrm{C}$ virus antibody were negative. Based on the presence of the dark ring in both eyes, the diagnosis of WD was suspected; therefore, further evaluation was conducted.

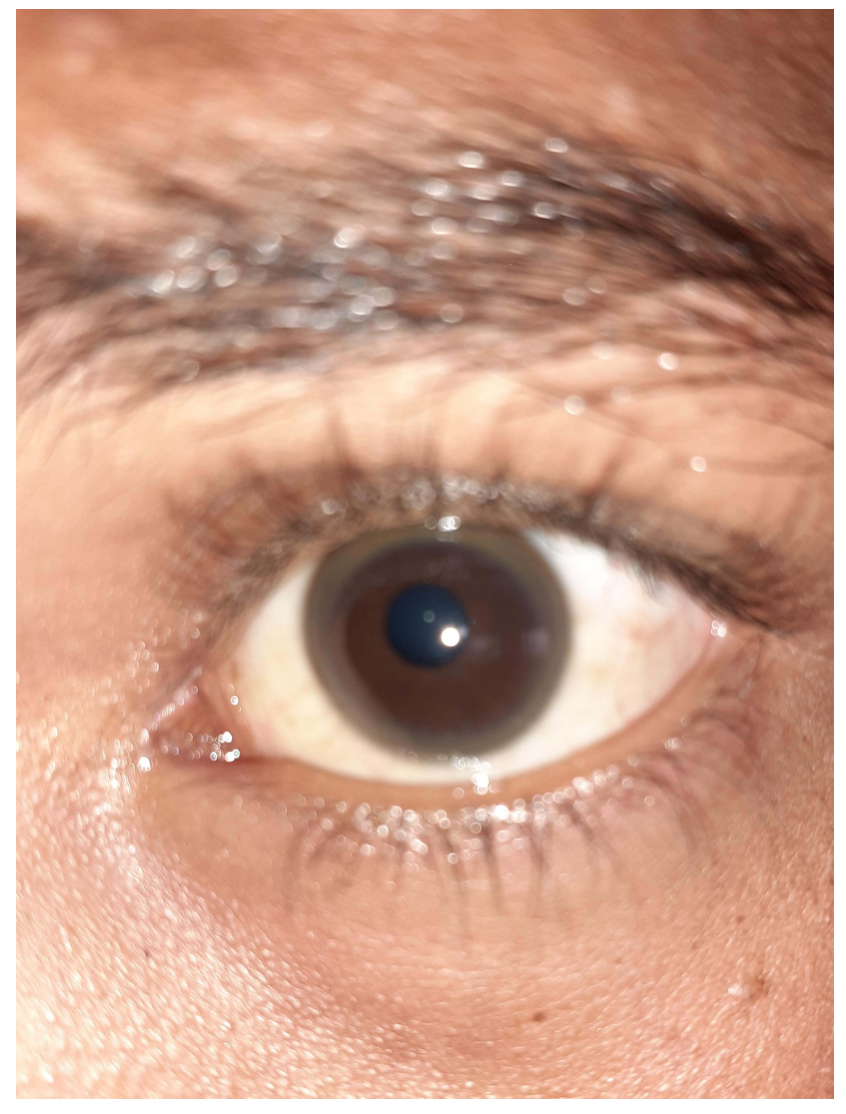

Figure I Kayser-Fleischer ring seen in the patient's left cornea.
Abdominal sonography revealed coarse echotexture of liver and cholelithiasis. Slit lamp examination confirmed the presence of Kayser-Fleischer (KF) ring in both eyes. Serum ceruloplasmin level was $0.10 \mathrm{~g} / \mathrm{L}$. Magnetic resonance imaging (MRI) which was done one year before showed mesial temporal sclerosis in the left temporal lobe. The patient's guardian was reluctant to do a followup MRI due to financial restriction.

Clinical characteristics, serum ceruloplasmin $(0.10 \mathrm{~g} /$ $\mathrm{L}$ ), and the KF rings on slit lamp examination were used to make the diagnosis of WD. According to the Ferenci score, a patient with a score of more than 4 is diagnosed as $\mathrm{WD}^{7}$

After confirmation of diagnosis, the treatment regimen was planned as follows: restriction of copper-rich foods, such as liver, mushroom, cocoa, chocolate, nuts, and shellfish; penicillamine $250 \mathrm{mg}$ once daily initially and increased up to $1.5 \mathrm{~g}$ /day in divided doses gradually; zinc acetate $150 \mathrm{mg}$ daily in three divided doses; pyridoxine $20 \mathrm{mg}$ daily; levetiracetam $500 \mathrm{mg}$ in two divided doses and increased in steps of $250 \mathrm{mg}$ every two weeks according to the patient's response; and propranolol $10 \mathrm{mg}$ three times per day. Six months after treatment, the patient had shown remarkable improvement, ie, was relieved of seizure attacks and was able to do her daily activities without assistance. The patient's parent provided written informed consent for the case details to be published.

\section{Discussion}

WD is an infrequent, genetic disorder of copper metabolism, in which the causative gene, ATP7B, results in dysfunction of the ATP7B transporter necessary for biliary excretion of copper and incorporation of copper into ceruloplasmin. ${ }^{8}$ More than 500 mutations have been identified in the ATP7B gene thus far. Even though there is some evidence that truncating mutations may be accompanied with earlier onset than missense mutations, and patients with frameshift mutations may be more likely to develop neurologic symptoms, individual gene mutations have not been found to accompany various manifestations of WD. Studies have reported cases of monozygotic twins with WD who were phenotypically discordant. This finding demonstrates that environmental and epigenetic factors might play a significant role in $\mathrm{WD}$, at least in part. ${ }^{9}$ WD usually starts with a clinically silent period, during which copper accumulation in liver results in subclinical hepatitis and progresses toward liver cirrhosis and appearance of neuropsychiatric manifestations. ${ }^{1}$ 
Changes in behavior, decline in academic performance, or inability to carry out activities that need strong hand-eye coordination may be found in pediatric patients insidiously in the early course of disease. Hand-writing may become worse, leading to cramped, small handwriting (micrographia) as seen in Parkinson's disease. Tremor, incoordination, excessive salivation, dysarthria, dystonia, and spasticity are all prevalent neurologic manifestations of WD. Transfer dysphagia may occur as a result of pseudobulbar palsy, with the risk of aspiration in severe cases. Dysautonomia, migraine headaches, and sleeplessness may also be present, although seizures are uncommon. ${ }^{10}$

Rarely, epileptic seizures can be the presenting symptom of WD, which can happen at any stage of the disease and might be more usual after initiation of anticoppering treatment. ${ }^{11,12}$ Seizures can affect $14.5 \%$ of patients with neurologic WD, particularly those who have cortical, subcortical, or cerebellar involvement on MRI. ${ }^{13}$ Various types of seizures such as grand mal, focal, or absence can be present. ${ }^{14}$ The types of seizures seen in WD patients do not differ significantly from those observed in general population surveys, ${ }^{4}$ although there is an increase of focal motor seizures in WD. ${ }^{4}$ Diseases characterized by involuntary and paroxysmal movements can mimic epilepsy. Detecting the presence or absence of seizures can be challenging in such conditions. $^{4}$ Epilepsy related to WD appears to have a better outcome than epilepsy in the general population. ${ }^{4}$ The prognosis of epilepsy appears to be influenced more by WD therapy than by anticonvulsants. $^{4}$ Treatment of such epilepsy cases is conventional like other forms, but treatment of Wilson disease must include penicillamine or trientine. ${ }^{14}$ When choosing antiepileptic medications for WD patients, their possible hepatotoxicity should be considered (as such, treatment with valproate should be avoided). ${ }^{3,4}$ Cerebellar signs and symptoms are rarely clinically relevant and are not found alone. On examination, frank limb ataxia is unusual. Cerebellar signs, apart from limb dysmetria such as overshoot dysmetria of the eyes and extremities, or ataxic dysarthria can be seen. ${ }^{15}$

WD is diagnosed based on some clinical and biochemical tests. Age at onset between 5 and 40 years, detectable $\mathrm{KF}$ rings, and decreased serum ceruloplasmin are the classic manifestations of WD. ${ }^{16} \mathrm{KF}$ rings caused by copper accumulation in Descemet's membrane, a pathognomonic sign, may be detectable in the eyes, either directly or on slit lamp examination. ${ }^{17}$

Initial presentations of WD are easily ignored and often diagnosed incorrectly as hepatitis, cirrhosis, splenomegaly, or encephalitis, leading to postponed therapy. ${ }^{18}$ The average time between development of symptoms and diagnosis is about two years. This delay is longer in neurologic than hepatic presentations, according to Kim et al (44 vs 14 months). ${ }^{18}$ A delay of 30 years has also been reported. ${ }^{19}$ Providing timely diagnosis and proper management may prevent irreversible organ damage. ${ }^{18}$ Pharmacotherapy of WD focuses on de-coppering through chelators and/or zinc to decrease the intestinal absorption of copper. ${ }^{8}$ Drug therapy in WD must be lifelong as abnormal copper deposition cannot be controlled by a lower copper diet. ${ }^{11}$ In patients with neurological features, clinical improvement begins 5-6 months after beginning de-coppering treatment, and most patients finally demonstrate significant improvement. ${ }^{8}$ Paradoxically, the neurological symptoms are said to be aggravated with penicillamine. This may be ascribed to mobilization of copper from liver and elevations of unbound copper levels which result in deteriorating of neurological manifestations. Some studies have reported an initial neurological worsening in 30-75\% of patients after penicillamine treatment. However, this has been denied by some other reports. ${ }^{20}$ The neurologic symptoms ameliorated in our patient after treatment with penicillamine.

WD being an inherited disorder, it is highly recommended to carry out familial screening in WD patients. The American Association for the Study of Liver Diseases (AASLD) and the European Association for the Study of Liver (EASL) suggest screening the first-degree relatives of a proband. First-degree relatives not only include the siblings of a proband, but also the children and parents. ${ }^{21}$ Our patient presented with unusual features such as generalized tonic-clonic epilepsy and predominant cerebellar findings. Asymptomatic liver changes were noted on abdominal sonography. She was misdiagnosed as encephalitis and epilepsy for one year. After following WD treatment for 6 months, she had noticeable improvements. This case highlights the importance of suspecting WD in young patients presenting with unknown isolated neurological signs such as seizure or cerebellar dysfunction. Eye examination, either directly or by slit lamp, and abdominal sonography (even in the absence of clinical evidence of hepatic involvement) in patients with movement disorders can help to diagnose life-threatening diseases. Likewise, 
on-time diagnosis and management of WD could help patients enjoy a healthy lifestyle during the rest of their lives.

\section{Ethics}

This report was approved by the Ethics Committee of the Department of Neuropsychiatry, Kabul University of Medical Sciences under protocol no. 2020-130.

\section{Funding}

There is no funding to report.

\section{Disclosure}

The authors report no conflicts of interest for this work.

\section{References}

1. Güngör Ş, Selimoğlu MA, Varol Fİ, et al. Pediatric Wilson's disease: findings in different presentations. A cross-sectional study. Sao Paulo Med J. 2018;136(4):304-309. doi:10.1590/1516-3180.2018.02102 30718

2. Žigrai M, Vyskočil M, Tóthová A, et al. Late-onset Wilson's disease. Front Med. 2020;7(26). doi:10.3389/fmed.2020.00026.

3. Członkowska A, Litwin T, Chabik G. Wilson disease: neurologic features. Handb Clin Neurol. 2017;142:101-119.

4. Dening TR, Berrios GE, Walshe JM. Wilson's disease and epilepsy. Brain. 1988;111(Pt 5):1139-1155. doi:10.1093/brain/111.5.1139

5. Dusek P, Litwin T, Członkowska A. Neurologic impairment in Wilson disease. Ann Transl Med. 2019;7:10.

6. Liu J, Luan J, Zhou X, et al. Epidemiology, diagnosis, and treatment of Wilson's disease. Intractable Rare Dis Res. 2017;6(4):249-255. doi:10.5582/irdr.2017.01057

7. Litwin T, et al. Psychiatric manifestations in Wilson's disease: possibilities and difficulties for treatment. Ther Advan Psychopharmacol. 2018;8:199-211.
8. Kathawala M, Hirschfield GM. Insights into the management of Wilson's disease. Therap Adv Gastroenterol. 2017;10(11):889-905. doi:10.1177/1756283X17731520

9. Mulligan C, Bronstein JM. Wilson disease: an overview and approach to management. Neurol Clin. 2020;38(2):417-432. doi:10.1016/j.ncl.2020.01.005

10. Roberts EA, Schilsky ML. Diagnosis and treatment of Wilson disease: an update. Hepatology. 2008;47(6):2089-2111. doi:10.1002/ hep. 22261

11. Bandmann O, Weiss KH, Kaler SG. Wilson's disease and other neurological copper disorders. Lancet Neurol. 2015;14(1):103-113. doi:10.1016/S1474-4422(14)70190-5

12. Cao C, Colangelo T, Dhanekula RK, et al. A rare case of wilson disease in a 72-year-old patient. ACG Case Rep J. 2019;6(3):e00024. doi: $10.14309 / \mathrm{crj} .0000000000000024$

13. Kalita J, Misra UK, Kumar V, et al. Predictors of seizure in Wilson disease: a clinico-radiological and biomarkers study. Neurotoxicology. 2019;71:87-92. doi:10.1016/j.neuro.2018.12.005

14. Walshe JM. Wilson disease. In: Andermann F, Guerrini R, Shorvon SD, editors. The Causes of Epilepsy: Common and Uncommon Causes in Adults and Children. Cambridge: Cambridge University Press; 2011:249-251.

15. Lorincz M. Neurologic Wilson's disease. Ann N Y Acad Sci. 2010;1184:173-187. doi:10.1111/j.1749-6632.2009.05109.x

16. Cao C, Colangelo T, Dhanekula RK, et al. A rare case of Wilson disease in a 72-year-old patient. ACG Case Rep J. 2019;6(3):1.

17. Pradeepkumar S, Rudrappa R, Rajakumar S. Neuro Wilson's - an Alien Presentation. Ann Int Med Dental Res. 2016;3.

18. Kim M-K, Lee K, Woo H-Y, et al. Late diagnosis of wilson disease, initially presenting as cerebellar atrophy mimicking spinocerebellar ataxia, by multigene panel testing. Ann Lab Med. 2020;40 (6):500-503. doi:10.3343/alm.2020.40.6.500

19. Shribman S, Warner TT, Dooley JS. Clinical presentations of Wilson disease. Ann Transl Med. 2019;7:6.

20. Kaur H, Kaur K, Sharma N, Kumar K. Wilson's disease: a case report. International Journal of Contemporary Medical Research. 2019;6(7):G42-44.

21. Woimant F, Djebrani-Oussedik N, Poujois A. New tools for Wilson's disease diagnosis: exchangeable copper fraction. Ann Transl Med. 2019;7:16
International Medical Case Reports Journal

\section{Publish your work in this journal}

The International Medical Case Reports Journal is an international, peer-reviewed open-access journal publishing original case reports from all medical specialties. Previously unpublished medical posters are also accepted relating to any area of clinical or preclinical science. Submissions should not normally exceed 2,000 words or 4

\section{Dovepress}

published pages including figures, diagrams and references. The manuscript management system is completely online and includes a very quick and fair peer-review system, which is all easy to use. Visit http://www.dovepress.com/testimonials.php to read real quotes from published authors. 